\title{
The Second Hit of Fatal Pneumonia by Cytomegalovirus in a Patient With Human Immunodeficiency Virus Infection
}

\author{
Hsin-Ju Tang, ${ }^{1}$ Huwi-Chun Chao, ${ }^{2}$ and Wen-Liang $\mathrm{Yu}^{2,3,}{ }^{*}$ \\ ${ }^{1}$ Departments of Nursing, Chi-Mei Medical Center, Tainan City, Taiwan \\ ${ }^{2}$ Intensive Care Medicine, Chi-Mei Medical Center, Tainan City, Taiwan \\ ${ }^{3}$ Department of Medicine, Taipei Medical University, Taipei City, Taiwan \\ "Corresponding author: Wen-Liang Yu, Intensive Care Medicine, Chi-Mei Medical Center, Tainan City, Taiwan. Tel: +886-62812811, Fax: +886-62833351, E-mail: \\ Yuleon_md@yahoo.com.tw
}

Received 2016 February 02; Accepted 2016 February 03.

Keywords: Human Immunodeficiency Virus, Pneumocystis, Pneumonia, Cytomegalovirus

\section{Dear Editor,}

Pneumocystis pneumonia (PCP) is the most common opportunistic infection in patients with the human immunodeficiency virus (HIV). The most common manifestations of PCP are subacute onset of progressive dyspnea, fever, non-productive cough, chest discomfort and respiratory failure (1). While cytomegalovirus (CMV) infections with retinitis and gastrointestinal disease were most often observed in patients with HIV, CMV pneumonitis was rarely emphasized $(2,3)$. Negligence of CMV pneumonia could lead to serious consequences (4). Herein is reported an HIV+ case with second hit of fatal pneumonia to alert clinicians to the potentially high mortality of CMV pneumonitis in the patients with HIV.

A 27-year-old young male with severe kyphoscoliosis had cough and fever for two weeks. Hence, he was brought to the hospital, where chest x-ray (CXR) film showed mixed alveolar and interstitial infiltration predominantly over left lung field, probably pneumonia (Figure 1A). Rotatory scoliosis of thoracic spine with convexity to right side was noticed. He had no other systemic diseases. He was intubated due to acute respiratory failure. Laboratory data included a white blood cell (WBC) count of 5,200/ $\mu \mathrm{L}$; monocyte, 4.8\%; hemoglobin, $9.2 \mathrm{~g} / \mathrm{dL}$; platelet count, $180,000 / \mu \mathrm{L}$; C-reactive protein (CRP), $80.1 \mathrm{mg} / \mathrm{L}$; procalcitonin, $3.52 \mathrm{ng} / \mathrm{mL}$ and albumin, $1.7 \mathrm{~g} / \mathrm{dL}$. An anti-HIV antibody was positive and CD4+ lymphocyte count was $12 / \mu \mathrm{L}$ with a CD4+/CD8+ ratio of 0.18. The HIV viral load was 66,321 copies/mL. The patient was admitted to the intensive care unit (ICU) with antibiotic therapy including intravenous levofloxacin (750 mg, on prescription), tigecycline (50 mg, every 12 hours) and trimethoprim/sulfamethoxazole (1200/240 mg, every six hours). The sputum, urine and blood cultures showed no bacterial growth. The clinical condition greatly improved and CXR film showed nearly complete resolution of the lung infiltration after one week of therapy (Figure 1B). The procalcitonin level dropped to $0.48 \mathrm{ng} / \mathrm{mL}$. However, the second hit of pneumonia accompanied by septic shock developed on the day 11 of the hospitalization, followed by rapid clinical deterioration without response to the same antibiotic regimen. Follow-up CXR film showed worsening infiltrates over left lung field (Figure $1 \mathrm{C}$ ). The WBC count was 3,000/ $\mu \mathrm{L}$; band, $13.0 \%$; monocyte, $13.0 \%$; platelet count, 21,000/uL; CRP, $130.0 \mathrm{mg} / \mathrm{L}$ and procalcitonin, $48.21 \mathrm{ng} / \mathrm{mL}$. An arterial blood gas showed hypoxemia with pH, 7.319; $\mathrm{PCO} 2,33.0 \mathrm{mmHg}$; $\mathrm{PO}$, 67.9 mmHg; base excess, $-7.5 \mathrm{mM} / \mathrm{L}$ and $\mathrm{PaO}_{2} / \mathrm{FiO}_{2}$ ratio, 150.9 $\mathrm{mmHg}\left(\mathrm{FiO}_{2}, 45 \%\right)$. Meanwhile, hemodynamic support could not rescue the patient. The family decided to have palliative therapy and the patient died on the day 13 of the hospitalization. The results of CMV-polymerase chain reaction for sputum and blood samples obtained on day 12 were both positive. Parental ganciclovir was not given in time.

In this HIV case, empirical therapy against initial pneumonia suspicion for PCP and usual bacterial pathogens achieved a good therapeutic response. However, subsequent CMV reactivation was detected in the sputum and blood samples during the second hit of recurrent pneumonia. The course was rapidly deteriorated without response to previous regimen, thus suggesting that CMV pneumonia rather than PCP resulted in the fatal event. Although CMV pneumonitis usually receives less attention than PCP, this case might highlight the necessity of early diagnosis and therapy for CMV infection in patients with HIV, especially with the second hit of pneumonia subsequent to successful therapy for PCP. 
Figure 1. CXR Film Shows Mixed Alveolar and Interstitial Infiltration Predominating
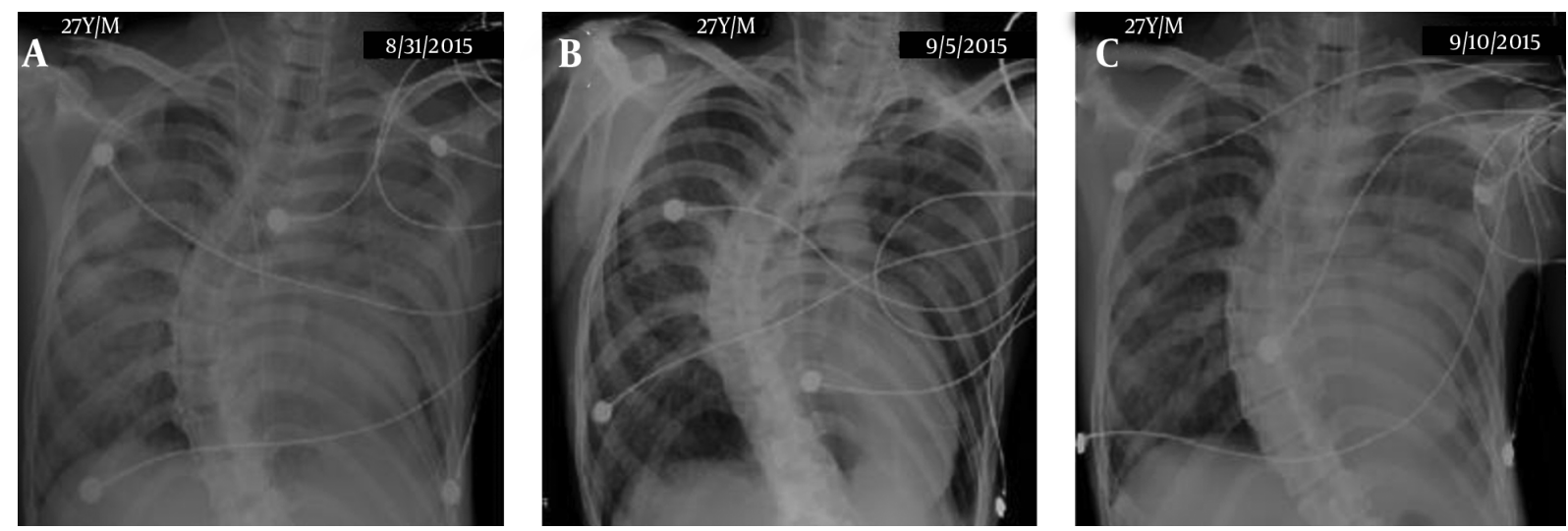

A, Left lung field; B, nearly complete resolution of the lung infiltration; C, recurrent infiltrates over left lung field.

\section{Acknowledgments}

None declared.

\section{Footnotes}

Authors' Contribution: Wen-Liang Yu: The guarantor, the original idea and protocol development, data abstracting and analyzing, and writing the manuscript; Hsin-Ju Tang and Huwi-Chun Chao, Protocol development, data abstracting and preparing the manuscript.

Conflict of interest: None declared.

Funding/Support: None declared

\section{References}

1. Huang L, Crothers K. HIV-associated opportunistic pneumonias. Respirology. 2009;14(4):474-85. doi: 10.1111/j.1440-1843.2009.01534.x. [PubMed: 19645867].

2. Osawa R, Singh N. Cytomegalovirus infection in critically ill patients: a systematic review. Crit Care. 2009;13(3):R68. doi: 10.1186/cc7875. [PubMed: 19442306].

3. Rodriguez-Barradas MC, Stool E, Musher DM, Gathe JJ, Goldstein J, Genta RM, et al. Diagnosing and treating cytomegalovirus pneumonia in patients with AIDS. Clin Infect Dis. 1996;23(1):76-81. [PubMed: 8816133].

4. Su MY, Tan CK, Yu WL. Difficult-to-treat, life-threatening cytomegalovirus pneumonia in a patient with chronic renal disease. Int J Infect. 2016;In Press(In Press) doi: 10.17795/iji-35300. 